\title{
A Parallel Framework for Real-time Dehazing
}

\author{
Yue Cheng ${ }^{123 *}$, Pengcheng Wen ${ }^{12 a}$, Lei Zhang ${ }^{12 b}$ and Zhengjun Zhai ${ }^{3 c}$ \\ ${ }^{1}$ AVIC Computing Technique Research Institute, Xian 710065, China \\ ${ }^{2}$ Aviation Key Laboratory of Science and Technology on Airborne and \\ Missileborne Computer, Xi'an, 710065, China \\ ${ }^{3}$ School of Computer Science, Northwestern Polytechnical University, Xi'an, \\ 201800, China \\ "chengyue198552@163.com; ${ }^{a}$ wpcheng@avic.com; \\ bhangleiactri@163.com; ${ }^{c}$ zhaizjun@nwpu.edu.cn
}

\begin{abstract}
In this paper, a parallel framework for image dehazing alghorithm is proposed to accelerate the computation on multi-core platform. Firstly parallel $O(1)$ complexity local minimum filter is employed to get the initial dark channel image, which is further refined by parallel Joint Recursive Bilateral Filter. Combined with the atmosphere parameter which is obtained by histogram based estimation, the dehazing result is finally achieved. The proposed method is evaluated on multi-core PC platform using $C++$ programming language with OpenMP compiler directive. Experimental results show that the proposed method outperforms available state-of-the-art Guided Filter based method and has a realtime performance.
\end{abstract}

Keywords: Real-time Dehazing, JRBF, Multi-core, OpenMP

\section{Introduction}

The light is scattered by the small dust particles and little droplets in the air when passing through the atmosphere. These aerosols can reduce the direct scene transmission and make the captured image covered with an ambient light layer offering a limited visibility of the scene. It is called hazing effect in the photographs, which often occurs in hazy or dusty weather conditions and even takes place in relatively clear days but from long distance capturing. Image dehazing is then implemented to recover the clear image from fog, mist etc contaminated image.

In image processing field, the hazy image is commonly modeled as

$I=J t+A(1-t)$

where $I$ is the observed light intensity and $J$ is the scene radiance which is treated as haze-free image, $A$ is the global atmospheric light, and $t$ is the medium transmission describing the portion of the light that is not scattered in the air and received by the camera. Equation (1) holds for each pixel position $\mathbf{x}=[x, y]^{T}$ and color channel $C \in\{\mathrm{R}, \mathrm{G}, \mathrm{B}\}$ for image $I_{C}(\mathbf{x})$. The goal of haze removal is to recover scene radiance $J$ from haze-contaminated image $I$. It is commonly treated as an inverse problem. Thus some priors or assumptions need to be known in advance to solve the dehazing problem.

There are a lot of priors about the hazing model, among which dark channel prior is thought to be the best one. In this assumption, dark pixels will be found in a small neighborhood area of color channels of common pixel. The two step strategy is developed in He's framework $[1,2]$, including local minimum filtering and dark 
channel refining. It should be noted that the main computational cost of dehazing using dark-channel prior is in its refining procedure. In He's early work, softmatting is used to get the refined dark-channel image. The dehazing result is satisfactory, however, its computational cost is too large to real-time applications such as Enhanced Vision System in aviation field. In He's later work, Guided Filter is proposed to improve the computation efficiency which has a constant $\mathrm{O}(1)$ computational complexity. It should be pointed that in every pixel position Guided Filter has about 9 times division operations, thus the computational efficiency decreases significantly and real-time performance is not reached.

Recently, Yang et. al. [3] proposed a recursive bilateral filter. It's a numerical approximation of traditional bilateral filter except that the system function in Zdomain corresponds to a recursive system. So the filtered-output of current pixel is only related to current input pixel and previous filtered pixel. The excellent property of recursive filter makes calculation convenient and fast. Only three buffers including current input, previous output and current output are needed to get an approximate bilateral filtered image. And the computational complexity of recursive bilateral filter is exact $\mathrm{O}(1)$ without any division. It's noticeable that recursive bilateral filter has separable property, and makes the parallel acceleration possible and programming implement convenient. The promoting property of recursive bilateral filter gives us the opportunity of refining dark channel image in highefficiency way, and makes the dehazing obtain real-time performance.

In this paper, we mainly focus on the computational efficiency of dehazing method and propose a new parallel dehazing framework shown in Figure 1. The first step is obtaining the local minimum value in every pixels to get the initial dark channel value, which is further refined in the second step by parallel Joint Recursive Bilateral Filter (JRBF). Combined with the atmosphere parameter estimated in the third step, the dehazing result is finally achieved in the fourth step. The four steps in dehazing framework are transformed to their separable forms, the solving procedure then gains a parallel acceleration. With the OpenMP compiler directive being implemented, the most calculations of dehazing are running in multi-cores on CPU simultaneously and the final solution has a real time performance.

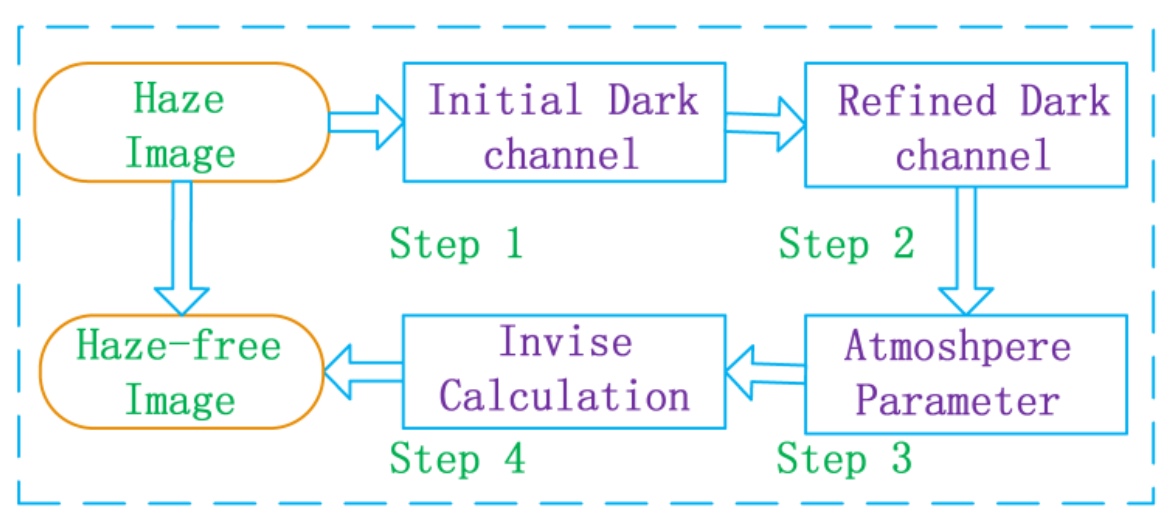

Figure 1. Schematic Diagram of the Dehazing Procedure

\section{Related Work}

Tan [4] observes that the haze-free image must have higher contrast compared with the input hazy image and he removes the haze component in the image by maximizing the local contrast of the restored image. Based on haze imaging model described in Equation (1), Tan's method treats medium transmission $t$ as a hint variable to enhance scene radiance $J$. The Markov Random Field (MRF) model of 
$J$ is further used to calculate $t$ by Graph Cuts or Belief Propagation [5] method. This approach is able to enhance the details of the haze-contaminated image. But the output image often fails in larger saturation area and has some obvious artifacts. Furthermore, the cost of calculation for solving MRF is normally very large and it is unacceptable for real-time applications.

Alternative Fattal [6] estimates the albedo of the scene and then infers the medium transmission under the assumption that the transmission and surface shading are locally uncorrelated. Fattal's method assumes the piecewise constant albedo in the haze-free image, and the direction of albedo vector can be estimated by Independent Component Analysis (ICA). As the MRF model is also used in Fattal's approach to get the final output, the cost of calculation is also large. It is noticeable that this approach is based on statistically independent assumption in a local image patch, so significant independent components are required in the patch to avoid the degenerate solution.

Recently Fattal [7] further proposes the color-lines prior for image dehazing. It assumes that small image patches typically exhibit a one-dimensional distribution in RGB color space. These color-lines in the context of hazy scenes are used to recover the medium transmission. The MRF model is also dedicated for producing complete and regularized transmission map. The improved model for dehazing physically sounds and gives better results. But the problem of calculation still exists and it can't be used in real-time applications.

He $[1,2]$ proposed a new simple prior to infer the transmission, locally, from dark-channel pixels found within a small neighborhood area. Based on the observation on many haze-free outdoor images, it's found that at least one color channel has very low intensity at some pixels. Thus there exists a prior about hazefree image that those pixels with at least one dark channel can be found nearby holds in many regions of the image, even there are large regions where only bright pixels are available. This approach has a very simply calculation form and can produce impressive dehazing results. However, it often has some artificial effect in large sky area.

Improvements for dark channel prior have been proposed [7-11]. Among these efforts, much attention is mainly paid on the haze removal results, the computing efficiency is not considered enough. It should be noted that the key step in dark channel dehazing is the estimation of the refined transmission map to further calculate the haze-free image. The early approach employs soft-matting, which is quite time-consuming. A improved approach uses the $\mathrm{O}(1)$ complexity Guided Filter [8], to largely speed up the dehazing procedure, where the Guided Filter is used as the joint filter in dehazing. The filter parameters are extracted in input hazy image and the filter target is the transmission image. So the refined transmission image has the similar texture of the input hazy image. However, the real-time performance for Guided Filter based method has not been reached.

Another fast image filter called recursive Bilateral Filter is proposed by Yang [3]. It is a numerical approximation of traditional Bilateral Filter with the transfer function in Zdomain corresponding to a recursive system. So the filtered output of current image pixel is only related to current input and previous output one. The excellent property of recursive filter makes the calculation convenient and fast. Moreover the recursive Bilateral Filter has separable property which makes the two dimensional filter operation convert to a one dimensional horizontal filter and a successive one dimensional vertical filter. Thus the recursive Bilateral Filter has the potential to speed up dehazing calculation in the parallel framework and has less computing time than Guided Filter when it is used as joint filter. 


\section{Proposed Method}

Using the dark channel prior, the transmission $t$ for each pixel position $\mathbf{x}$ can be calculated by

$$
t(\mathbf{x})=1-\frac{\min _{\{c, \mathbf{y}\}}\left\{I_{C}(\mathbf{y})\right\}}{A_{\mathrm{M}}}=1-\frac{D(\mathbf{x})}{A_{\mathrm{M}}},
$$

where $\mathbf{y} \in \Omega(\mathbf{x})$ is the neighborhood pixel position of $\mathbf{x}, D(\mathbf{x})$ is the dark channel in pixel position $\mathbf{x} . A_{\mathrm{M}}$ is the mean value for three channel atmosphere parameters $A_{C}$. The haze-free image can be solved using transmission and atmosphere parameters. Since a large dark channel value leads to a small transmission, the calculation in large sky area of the image will degenerate which brings some artificial effects in the result image. Thus a special threshold on $t$ is necessarily to be used to avoid the degenerate results in large sky area. In the proposed framework, we put equation (2) into equation (1). The haze-free image $J$ will be calculated by dark channel $D$ and atmosphere parameter $A_{C}$, thus

$$
J_{C}=\frac{A_{M} I_{C}-D A_{C}}{A_{\mathrm{M}}-D} .
$$

For input color image, the initial dark channel is calculated from local RGB minimum value. Firstly, we calculate the pixel-wise RGB minimum of hazy image as

$$
I_{\min }=\min _{C}\left(I_{C}\right)
$$

According to Van Herk's fast local minimum method [12], the two dimension local minimum filter in a box region can be separated to a linear row filter and a successive column filter. Considering an input one dimension signal $f(x)$ as the row of image, we need to calculate the minimum value in local region $[x-r, x+r]$, where $r$ is the filter radius in a box region. Firstly the forward comparison buffer can be calculated by

$$
g(x)=\left\{\begin{array}{cc}
f(x), & x=n k \\
\min (g(x-1), f(x)), & x \neq n k
\end{array},\right.
$$

where $n=(0,1,2, \ldots)$ and $k=2 r+1$ is the filter diameter. Then the backward comparison buffer is

$h(x)=\left\{\begin{array}{cc}f(x), & x=n k+k-1 \\ \min (h(x+1), f(x)), & x \neq n k+k-1\end{array}\right.$.

And the local minimum buffer is finally acquired by 
$s(x)=\min (h(x+r), g(x-r))$.

Each minimum row filter can be achieved by just three comparing operations for each pixel. The total two dimension local minimum filter has just six comparing operations for each pixel. What's more, the minimum filter can be achieved in a parallel framework by sending each row or column to different computing units. Then the initial dark channel image $D_{\text {init }}(\mathbf{x})$ is solved by finding local minimum of $I_{\min }(\mathbf{x})$ using the parallel computation.

Instead of refining transmission, now we directly refine the initial dark channel image. The refined dark channel image $D_{\text {refined }}(\mathbf{x})$ is calculated by joint Bilateral Filter as

$D_{\text {refined }}(\mathbf{x})=\sum_{\mathbf{y} \in \Omega(\mathbf{x})} R(\mathbf{y}) S(\mathbf{y}) D_{\text {init }}(\mathbf{y})$

where $R$ is the range parameter and $S$ is the space parameter. Both of these two parameters are extracted from the input hazy image. Thus the filter is used as a joint filter and the refined dark channel image has the similar texture details of input hazy image. The original Bilateral Filter has very computation complexity and hard to be used in real-time applications. So we use its approximation recursive form for the joint filter.

Firstly we use the one-dimension Bilateral Filter to introduce the recursive filter. The n-order causal system for input signal $x$ and output signal $y$ in time domain could be expressed as

$y_{i}=\sum_{l=0}^{n-1}\left(a_{l} \cdot x_{i-1}\right)-\sum_{k=1}^{n}\left(b_{k} \cdot y_{i-k}\right)$

where $a$ and $b$ are recursive parameters. After applying Z-transform to both sides of equation (9) shown as below

$Y(Z)=\sum_{l=0}^{n-1}\left(a_{l} \cdot X(Z) Z^{-l}\right)-\sum_{k=1}^{n}\left(b_{k} \cdot Y(Z) Z^{-k}\right)$.

This recursive system is then characterized by the following transfer function

$$
\begin{aligned}
H^{a}(Z) & =\frac{\sum_{l=0}^{n-1} a_{l} \cdot Z^{-l}}{1+\sum_{k=1}^{n} b_{k} \cdot Z^{-k}} \\
& =\sum_{k=0}^{+\infty} h_{k}^{a} \cdot Z^{-k},
\end{aligned}
$$

where $\left\{h_{k}^{a}\right\}$ denote the impulse response of the recursive system whose Z-transform is $H^{a}(Z)$. When a special system function $H(Z)$ is specified with impulse response 
$\left\{h_{k}\right\}$, to get the approximation system of $H^{a}(Z), \hat{a}_{l}$ and $\hat{b}_{k}$ are estimated by the error function

$\left(\hat{a}_{l}, \hat{b}_{k}\right)=\arg \min \left(\sum_{k=0}^{+\infty} h_{k}^{a}-h_{k}\right)^{2}$

According to [3], the simplest recursive bilateral system is

$y_{i}=(1-a) \cdot x_{i}+R_{i, i-l} \cdot a \cdot y_{i-1}$,

where $a \in(0,1)$ is the feedback space parameter and

$R_{i, i-l}=\exp \left(-\frac{\left|x_{i}-x_{i-1}\right|^{2}}{2 \sigma_{R}^{2}}\right)$

is the recursive range parameter. With minor modification, the recursive gradient domain bilateral system is

$y_{i}=\left(1-R_{i, i-1} \cdot a\right) \cdot x_{i}+R_{i, i-1} \cdot a \cdot y_{i-1}$.

It is proved in [3] that gradient domain recursive Bilateral Filter has better performance in detail preservation. And the recursive filter has separation property. We just use it twice for rows and columns of $D_{\text {init }}(\mathbf{x})$ successively, the $D_{\text {refined }}(\mathbf{x})$ will be filtered in the two dimensions. Because the two linear recursive filters can be sent to different computing units, the refinement of dark channel image has totally parallelly speeded up.

In the atmosphere transmission model, each color channel has a global transmission parameter. The parameter is extracted from the special regions of hazy image where the corresponding dark channel value is higher. Thus the dark channel threshold should be firstly calculated. In our framework the threshold is corresponding to the highest $0.1 \%$ dark channel values.

In the third step of dehazing, we calculate the histogram $\operatorname{hist}(i), i \in(1,2, \ldots, 255)$ for dark channel image $D_{\text {refined }}(\mathbf{x})$. Then the threshold of dark channel is calculated by

$T=\arg \left(\sum_{T<i \leq 255} \operatorname{hist}(i)>N\right)$,

where $N$ is the count of $0.1 \%$ of the image size. Once the threshold has been obtained, the atmosphere parameter can be calculated as

$A_{C}=\frac{\sum I_{C}(x)}{N}, \quad$ s.t. $\quad D(\mathbf{x})>T$,

The calculation of the histogram for the dark channel image can be achieved by classical parallel reduction algorithm. It's unnecessary in our framework because the amount of the calculation is quite small. After extracting the dark channel and the 
atmosphere parameters, the dehazing result is extracted by Equation (3) in the final step.

As shown in above discussion, each step in the proposed framework could be decomposed in its corresponding parallel operations, the entire haze removal procedure gains a totally parallel acceleration.

\section{Experiment Results}

We evaluate the proposed method on Intel 2.2GHz i7-2720QM laptop platform with 4 cores and $8 \mathrm{G}$ memory. All methods are implemented with $\mathrm{C}++$ and OpenMP complier directive. The state-of-the-art method using Guided Filter is compared in the experiment.

The refinement of dark channel is the most time consuming part in the dehazing framework. So firstly we evaluate the performance of Guided Filter and proposed parallel Joint Recursive Bilateral Filter (JRBF) for dark channel refinement. The JRBF method is either running in single core or 4 cores of the same computer. The computing time is listed in Figure 2. It's obvious that Guided Filter has the longest processing time. For all resolutions of Guided Filter, the computing time is exceeded $100 \mathrm{~ms}$, which is unacceptable for real-time applications. In contrast when employing JRBF the processing time decreases dramatically. Especially when using 4-core JRBF, the processing time decreases significantly. It takes no more than $27 \mathrm{~ms}$ even for $1024 \times 768$ resolution initial dark image. Thus it is very suitable for real-time dehazing.

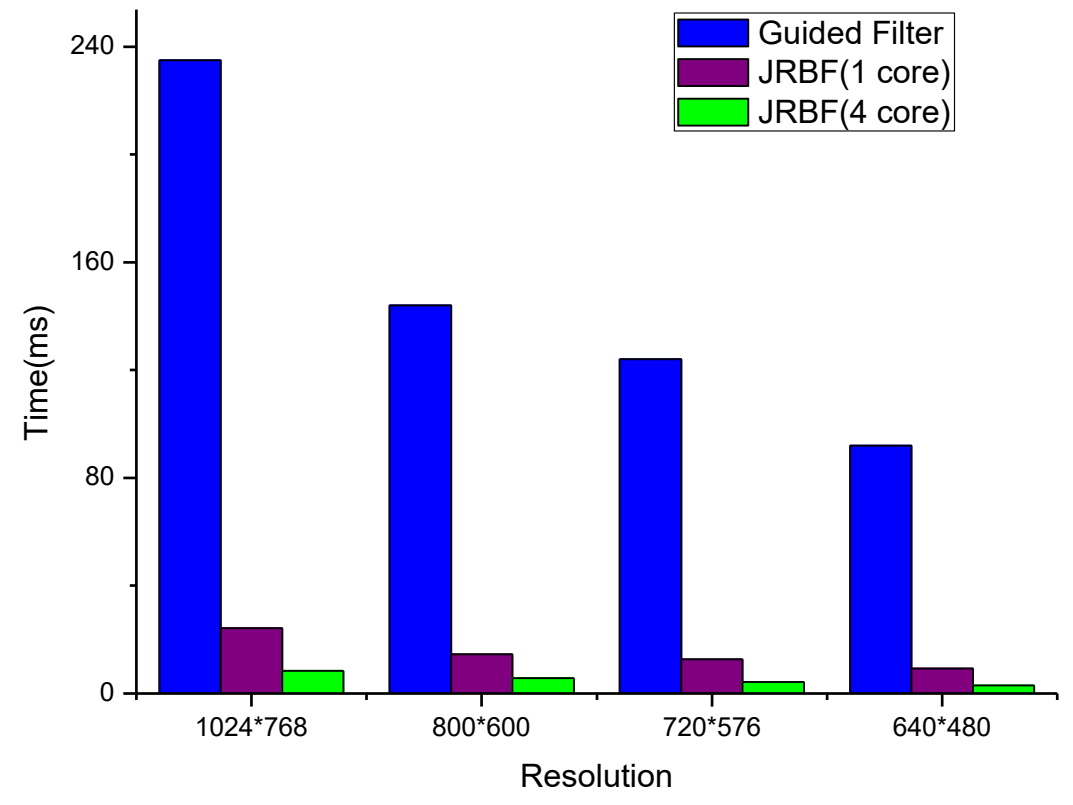

Figure. 2. Computing time for Different Initial Dark Channel Image using Different Joint Filters

Next we apply proposed four-step dehazing framework to different color hazy images. We selected one classic haze photograph (left), one image with large sky areas (middle) and one oversaturated picture to compare the dehazing effect of proposed method and Guided Filter based method. As shown in Figure 3, after dehazing with proposed method, the details on the image are enhanced. Although Guided Filter based method can improve image details in certain degree too, the proposed method gives a relative better estimation to the large sky areas contained in the middle and right photographs. As for the oversaturated picture (right), Guided Filter based method generates very distorted result of the overexposure part, while the proposed method gives a quit reliable image which is 
more similar to the original photograph. These suggest that proposed method is quite robust.
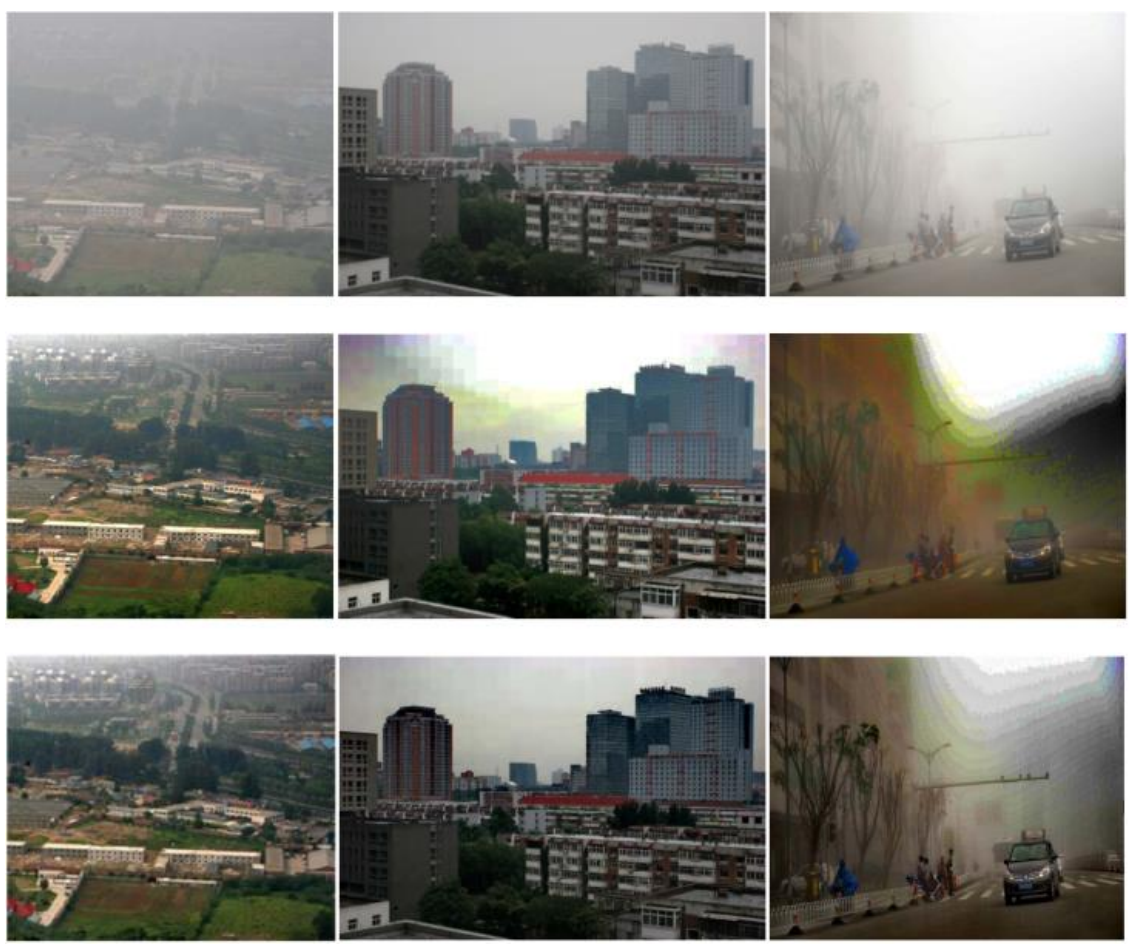

\section{Figure 3. Dehazing Results using Guided Filter method (second row) and Proposed Method (third row)}

Finally, we apply the proposed 4-steps dehazing frame for different resolution hazy images. Table 1 shows the multi-core processing time and acceleration ratios. The cost of $1024 \times 768$ and $720 \times 576$ resolution images are listed. The acceleration ratio is nearly linear to number of cores as shown in the table. Therefore it is proved that all steps in our framework gain definitely parallel acceleration.

\section{Table 1. Computing Time and Acceleration Ratios for Different Number of} Cores with Different Resolutions using Proposed Method

\begin{tabular}{ccccc}
\hline $\begin{array}{c}\text { Number } \\
\text { of Cores }\end{array}$ & \multicolumn{2}{c}{$\mathbf{1 0 2 4}$ *768 } & \multicolumn{2}{c}{$\mathbf{7 2 0 * 5 7 6}$} \\
\cline { 2 - 5 } & $\begin{array}{c}\text { Time } \\
(\mathrm{ms})\end{array}$ & $\begin{array}{c}\text { Acceleration } \\
\text { Ratios }\end{array}$ & $\begin{array}{c}\text { Time } \\
(\mathrm{ms})\end{array}$ & $\begin{array}{c}\text { Acceleration } \\
\text { Ratios }\end{array}$ \\
\hline $\mathbf{1}$ & 73.45 & 1.00 & 24.70 & 1.00 \\
\hline $\mathbf{2}$ & 39.78 & 1.85 & 13.43 & 1.84 \\
\hline $\mathbf{3}$ & 30.75 & 2.39 & 10.40 & 2.38 \\
\hline $\mathbf{4}$ & 26.07 & 2.82 & 8.87 & 2.78 \\
\hline
\end{tabular}

\section{Conclusion}

In conclusion, we present a new parallel framework for dehazing in this paper. The initial dark channel image and refined dark channel are obtained using parallel image filter. The atmosphere parameters are extracted in a parallel manner using the histogram based method. Finally the total framework gains a parallel acceleration. Experimental results show that the proposed method has the best performance among the state-of-theart Guided Filter based method both in the robustness and efficiency. For $1024 \times 768$ 
resolution color image, the processing time is less than $30 \mathrm{~ms}$ which fulfils the real-time requirement. We believe that the proposed method would have a better performance on GPU platform considering more number of computing units.

\section{Acknowledgments}

This work was supported by the AVIC Technology Innovation Foundation under Grant No. 2014D63130R and by the Aviation Science Foundation under Grant No. $2014 Z C 31004$.

\section{References}

[1] K. He, J. Sun and X. Tang, "Single image haze removal using dark channel prior", IEEE Conference on CVPR 2009, Miami, USA, (2009) June 19-26.

[2] K. He, J. Sun and X. Tang, "Single image haze removal using dark channel prior", IEEE Transactions on Pattern Analysis and Machine Intelligence, vol.33, no.12, (2011), pp. 2341-2353.

[3] Q. Yang, "Recursive bilateral filtering", 12th European Conference on Computer Vision, Florence, Italy (2012) October 7-13.

[4] R. T. Tan, "Visibility in bad weather from a single image", IEEE Conference on CVPR 2008, Anchorage, USA, (2008) June 23-28.

[5] C. M. Bishop, "Pattern recognition and machine learning", Springer, New York (2006).

[6] R. Fattal, "Single image dehazing", ACM Transactions on Graphics, vol. 27, no.3, (2008), pp. 72.

[7] R. Fattal, "Dehazing using Color-Lines", ACM Transaction on Graphics, vol. 34, no. 1, (2014), pp. 13

[8] K. He, J. Sun and X. Tang, "Guided image filtering", IEEE Transactions on Pattern Analysis and Machine Intelligence, vol. 35, no. 6 (2011), pp. 1397-1409.

[9] K. Gibson and T. Nguyen, "On the effectiveness of the dark channel prior for single image dehazing by approximating with minimum volume ellipsoids", Red 1, (2011).

[10] K. B. Gibson and T. Q. Nguyen, "Fast single image fog removal using the adaptive Wiener filter", 20th IEEE International Conference on Image Processing, Melbourne, Australia, (2013) Sep 15-18.

[11] K. B. Gibson and T. Q. Nguyen, "An analysis of single image defogging methods using a color ellipsoid framework", EURASIP Journal on Image and Video Processing (2013), pp. 1-14.

[12] M. Van Herk, "A fast algorithm for local minimum and maximum filters on rectangular and octagonal kernels”, Pattern Recognition Letters, vol.13, no. 7, (1992), pp. 517-521.
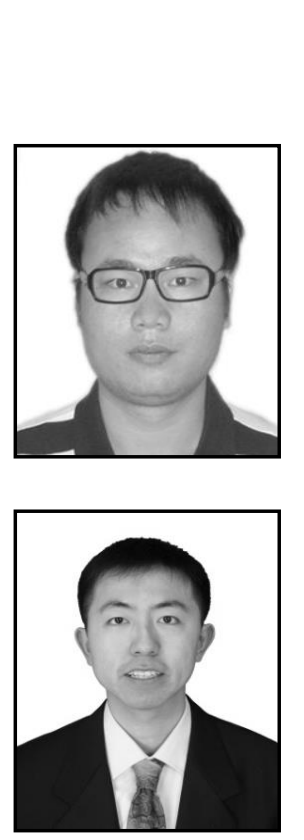

\section{Authors}

Yue Cheng, He received the B.S. and Ph.D. degree on Electronic Engineering from Zhejiang University in 2008 and 2013. Since 2013 he is working in AVIC Computing Technique Research Institute, Xi'an, where he is also a post doctor from 2014 and senior engineer from 2015. His research interests include Computer Vision and Augmented Reality in Airborne System.

Pengcheng Wen, He received his Ph.D. degree on instrument science and technology from Tianjin University in 2010. He was also a visiting scholar on computer science at University of Reading for one year (2008 - 2009). Now he is a senior AVIC Computing Technique Research Institute, Xi'an. His present research interest is image processing and intelligent computing. 

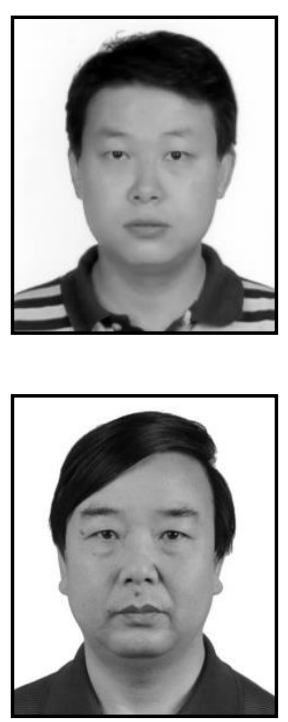

Lei Zhang, He received the B.S. degree in Computer Science from Xi'an Jiao Tong University in 2001, and MS. Degree from China Aviation Academe in 2011. Now he is working in Xi'an Aviation Computation Technique Research Institute, and he is also a PhD Candidate in Computer Science at Northwestern Polytechnical University. His research interests include Computer Vision and Image Processing.

Zhengjun Zhai, He received his B.S degree in Information System Engineering from Northwestern Polytechnical University (NPU) in 1985, where he also received M.S. degree in Computer Science and Technology in 1988. Now he is the director of Computer Measurement \& control and Simulation Technology Institute in NPU. His research interests include Information Processing, Embded System and Avionics System. 